J. Lake Sci. (湖泊科学), 2012, 24(3): 436-442

http: //www.jlakes.org. E-mail : jlakes@niglas.ac.cn

(C) 2012 by Journal of Lake Sciences

\title{
浙江金华江支流白沙溪水质硅藻生物监测方法"
}

\author{
李钟群 ${ }^{1}$, 袁 刚 $^{1 * *}$, 郝晓伟 ${ }^{1}$, 刘 威 ${ }^{2}$ \\ ( 1 : 浙江省水利科技推广与发展中心,杭州 310012) \\ $(2$ :珠江流域水环境监测中心,广州 510611)
}

\begin{abstract}
摘 要: 以白沙溪为示范区, 比较了硅藻生物指数评价与我国现阶段河流水质理化评价结果的异同性, 同时对白沙溪进 行水生态评估. 水质理化评价显示白沙溪水质从 $\mathrm{I}$ 类到劣 $\mathrm{V}$ 类均有出现, 而硅藻特定污染敏感指数和硅藻生物指数评价 白沙溪水质为 “优” 到 “差” 均有出现. 二者评价结果总体上相吻合, 同时亦存在一定差异. 硅藻生态类群组成显示前三个 断面以耐低污染硅藻、自养硅藻和喜好很高氧饱和度硅藻为主, 4 \#断面 (除 2010 年 11 月) 以耐中污染和强污染硅藻、异养 硅藻、喜好低氧硅藻类群占优势. 全年水体各断面均以喜中性和碱性的硅藻类群为主. 特定污染敏感指数和硅藻生物指 数均与电导率、总磷、氨氮、氯化物之间呈显著负相关, 此外硅藻生物指数还与高锰酸盐指数、总氮、亚硝酸盐氮和可溶性 磷酸盐之间呈显著负相关. 本研究结果对开展我国河流水质生物监测具有一定的借鉴意义, 但其在我国的适用性还需要 开展进一步的研究.
\end{abstract}

关键词: 白沙溪;硅藻;生物监测;水质;金华江

\section{Diatom-based biomonitoring method of water quality in Baisha River, a tributary of Jinhua River, Zhejiang Province}

\author{
LI Zhongqun ${ }^{1}$, YUAN Gang ${ }^{1}$, HAO Xiaowei ${ }^{1} \&$ LIU Wei ${ }^{2}$ \\ (1: Science and Technology Promotion Centre of Water Resources of Zhejiang Province, Hangzhou 310012, P. R. China) \\ (2: Environmental Monitoring Center of Pearl River Basin, Guangzhou 510611 ,P. R. China)
}

\begin{abstract}
Baisha River was chosen as a testing area in this research, and the similarities and differences were compared between diatom-based method and physico-chemical method in Chinese rivers at the present stage, besides, water ecological condition had also been assessed in Baisha River. The physico-chemical parameters showed that the water quality level of Baisha River is from Grade I to worse than Grade V, while Specific Pollution Sensitivity Index (SPI) and Biotic Diatom Index (BDI) indicated that its water quality is from bad to high quality level. These two assessment results were almost identical as a whole although differences between them did occur. For diatom eco-type, the results showed that the first three sites are principally dominated by oligosaprobous and $\beta$-mesosaprobous, N-autotrophic, Polyoxybiontic taxa, and the fourth site (except Nov. 2010) is principally dominated by Meso-to polysaprobous, N-heterotrophic and low aerobic diatom taxa. All sites were dominated by circumneutral and alkaliphilous species in Baisha River. There were negative significant relationships between SPI, BDI and conductivity, total phosphorus, ammonia nitrogen, and chloride. In addition, there were also negative significant relationships between $B D I$ and permanganate index, total nitrogen, nitrite nitrogen, and soluble phosphate. The results of the present study provide a good experience for biomonitoring in China, but more researches are needed in order to establish Chinese biomonitoring standard.
\end{abstract}

Keywords: Baisha River; diatom; biomonitoring; water quality; Jinhua River

硅藻是具有硅质骨架的单细胞藻类 ${ }^{[1]}$, 因其对河流营养盐浓度、pH 值、电导率、含氯度和重金属等变化 极敏感,故被认为是河流水环境可靠的生物指示种 ${ }^{[24]}$, 在判别水体污染程度、评价河流水环境富营养化状 态等方面具有广泛的应用价值 ${ }^{[5]}$. 用于评价河流水质的硅藻指数众多,包括 Specific Pollution Sensitivity In-

* 2011-07-21 收稿;2011-09-29 收修改稿. 李钟群, 男,1975 年生, 高级工程师; E-mail : lizq@ zjwater. gov. cn.

** 通信作者; E-mail : yuang@ zjwater. gov. cn. 
$\operatorname{dex}(S P I)^{[6]}$ 、Biotic Diatom Index $(B D I)^{[7]}$ 、Schiefele and Schreiner's Index $(S H E)^{[8]}$ 、Artois-Picardie Diatom In$\operatorname{dex}(A P D I)^{[9]}$ 、Watanabe Index $(W A T)^{[10]}$ 、Trophic Diatom Index $(T D I)^{[11]}$ 等. 其中应用最广泛的硅藻评价指标 有 $S P I$ 和 $B D I$, 这两个指数均可用来评价一个水域的生物质量, 监测一个水域生物质量的短暂变化和河流生 物质量的空间变化等 ${ }^{[12]}$. 欧美国家的一些水环境管理机构已经将硅藻应用于河流、湖泊的水质评价, 并取得 了较好的效果 ${ }^{[13-15]}$; 国内研究主要有硅藻群落与环境因子的相关性分析 ${ }^{[16-18]}$ 、指示性属种的选择 ${ }^{[19-20]}$ 以及 利用硅藻生物指数进行水生态状况分析, 关于前二者的报道较多, 而对于后者的报道较少, 如 Tang 等 ${ }^{[21]}$ 采 用 River Diatom Index $(R D I)$ 、赵湘桂等 ${ }^{[12]}$ 采用 $S P I 、 B D I$ 分别对香溪河和漓江进行了水生态状况评价.

白沙溪位于钱塘江一级支流金华江流域, 是当地的农牧业、水产业和工业的重要水源. 同时,白沙溪河 道水面开阔, 水位较低, 适合硅藻采样的基质较多, 符合河流硅藻样品采集的要求. 近年来, 随着工农业经济 的迅速发展, 大量水利工程的修建, 白沙溪水生态状况不断恶化. 因此亟需全面准确地评价白沙溪水生态状 况, 以期为其水生态修复提供基础资料和理论依据.

本研究选择浙江省金华江支流白沙溪作为硅藻监测示范区, 比较硅藻 $S P I 、 B D I$ 生物评价与我国河流水 质理化评价的异同性, 并进行白沙溪水生态状况评估, 以期为我国河流生态监测提供理论依据和实践参考, 为水资源保护和供水安全提供更为全面准确的资料.

\section{1 材料与方法}

\section{1 采样点及采样时间}

本研究分别于 2010 年 5 月、 2010 年 8 月、 2010 年 11 月和 2011 年 2 月采集硅藻样品,取样地位于 金华江支流白沙溪, $1^{\#}$ 断面位于白沙堰下游, $2^{\#}$ 断 面位于希能公司上游路口, $3^{\#}$ 断面位于新昌桥下 游, $4^{\#}$ 断面位于白龙桥下游 (图 1). 样点的选择兼 顾了白沙溪所受污染物类型的不同和污染梯度的

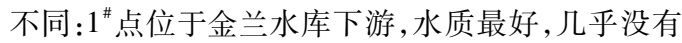
污染 $; 2^{\#}$ 点和 $3^{\#}$ 点水质次之, 主要受生活污水污染; $4^{\#}$ 点水质最差, 受工业废水和生活污水的双重 污染.

\section{2 采样方法}

硅藻采集方法根据法国 AFNOR（2000）T90$354^{[22]}$ 和欧洲 NF EN $13946^{[23]}$ 硅藻常规采样和预 处理指导标准. 硅藻采样的基质为处于地势开阔处 无树荫遮挡的活水区、硅藻生物膜可见且完全浸没 于水中的石块, 样品使用一次性牙刷刷取并置于样 品瓶中, 每个样点至少采集 5 块石块 (总面积约 $300 \mathrm{~cm}^{2}$ ), 混合样加甲醛固定. 现场测定水体 $\mathrm{pH}$ 值、电导率 (Cond)、溶解氧 ( DO) 、水温 ( WT) 等物 理参数, 并将水样带回实验室测定总磷 $(\mathrm{TP})$ 、总氮

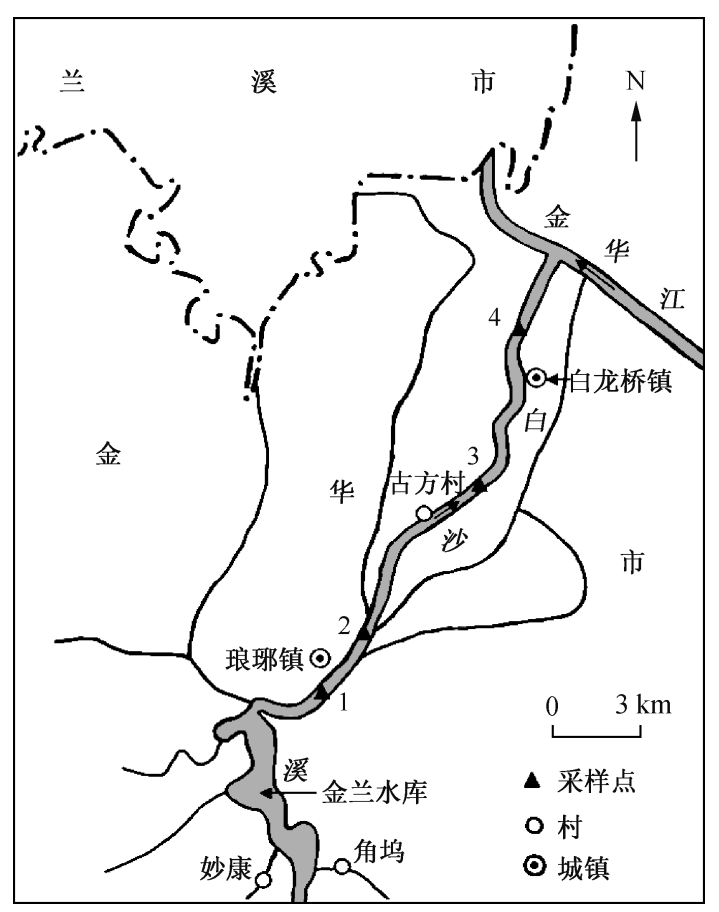

图 1 白沙溪采样点分布

Fig. 1 Distribution of sampling sites in Baisha River $(\mathrm{TN})$ 、高镇酸盐指数 $\left(\mathrm{COD}_{\mathrm{Mn}}\right)$ 、氯化物 $($ Chlo $)$ 、五日 生化需氧量 $\left(\mathrm{BOD}_{5}\right)$ 、铵氮 $\left(\mathrm{NH}_{4}^{+}-\mathrm{N}\right)$ 、硝酸盐氮 $\left(\mathrm{NO}_{3}^{-}-\mathrm{N}\right)$ 、亚硝酸盐氮 $\left(\mathrm{NO}_{2}^{-}-\mathrm{N}\right)$ 、可溶性磷 $(\mathrm{SDP})$ 、可溶性硅酸盐 $(\mathrm{SDSi})$ 等化学参数,监测方法依据《水环境监测规范》(SL219- 1998 $)^{[24]}$.

\section{3 硅藻镜检与计数}

将硅藻样品经双氧水和盐酸消解后, 用封片胶 (Naphrax) 封片, 在 $1000 \times$ 油镜下检出, 视野内所有的硅 藻样品及破损面积不超过 $1 / 4$ 的样品都要鉴定和计数, 计数总数超过 400 个以后, 计数结果用不同种的相对 丰度来表示.

\section{4 指数计算与水质评价}

$S P I 、 B D I$ 使用 OMNIDIA 软件 ${ }^{[25]}$ 计算, 河流水质硅藻生物评价标准为: $S P I 、 B D I \geqslant 17$, 优; $S P I<17 、 B D I \geqslant$ 
13 , 良; $S P I<13 、 B D I \geqslant 9$, 中; $S P I<9 、 B D I \geqslant 5$, 差; $S P I 、 B D I<5$, 很差 ${ }^{[22]}$. 理化水质状况分析依据《地表水环境 质量标准》( GB3838-2002), 采用单因子评价法进行, 其中参与评价项目包括 $\mathrm{pH} 、 \mathrm{DO} 、 \mathrm{COD}_{\mathrm{Mn}} 、 \mathrm{BOD}_{5}$ 、 $\mathrm{NH}_{4}^{+}-\mathrm{N} 、 \mathrm{TP}^{[26]}$.

\section{5 硅藻生态类群划分}

Van 等以水体酸碱度、溶解氧、盐度、N-自养性、耐有机污染程度等划分为不同的生态类群 ${ }^{[27]}$. 由于我国 缺乏对河流硅藻生态类群的系统划分,故本研究采用 Van Dam 硅藻生态类群划分方法.

\section{2 结果}

\section{1 硅藻组成及优势种}

共鉴定出 92 种硅藻 (含变型和变种), 物种数较为丰富. 其中相对丰度最高的三种硅藻为 Achnanthidium minutissimum (21.8\% )、A. rivulare(21.1\%) 和 Gomphonema incognitum(7.9\%). Nitzschia、Navicula、Fragilaria 和 Gomphonema 4 个属的物种数最多, 分别为 12 种、10 种、8 种和 8 种. 在所有断面均出现的硅藻种仅有 $A$. minutissimum. 各断面的物种丰富度分布范围为 $43 \sim 54$ 种, 均值为 47 种.

\section{2 理化指标和硅藻指标水质评价比较}

水质理化指标总体评价白沙溪水质从 $\mathrm{I}$ 类到劣 $\mathrm{V}$ 类均有出现, 而硅藻 $S P I$ 和 $B D I$ 指数评价白沙溪水质 为 “优” 到 “差” 均有出现 (表 1). 总体上看, 理化指标与硅藻指标水质评价结果总体上是吻合的. 对于不同的 监测断面, 水质理化评价和 $S P I$ 及 $B D I$ 生物评价亦有一定的差异: 如 2010 年 5 月, 水质理化评价 $2^{\#}$ 点为 III 类水,而 $S P I$ 和 $B D I$ 显示该水体均为 “优”;2010 年 11 月,水质理化评价 $2^{\#}$ 点为 IV 类水,而 $S P I$ 和 $B D I$ 显示 该水体为“优”和“良”. SPI 和 $B D I$ 之间存在极显著线性正相关 $(r=0.923, P<0.001)$ (表 2).

表 1 白沙溪水质理化与硅藻综合评价比较

Tab. 1 Comparison of water quality assessments between physico-chemical and diatom-based method in Baisha River

\begin{tabular}{|c|c|c|c|c|c|c|c|c|c|c|c|c|}
\hline \multirow{2}{*}{ 监测断面 } & \multicolumn{3}{|c|}{2010 年 5 月 } & \multicolumn{3}{|c|}{2010 年 8 月 } & \multicolumn{3}{|c|}{2010 年 11 月 } & \multicolumn{3}{|c|}{2011 年 2 月 } \\
\hline & $S P I$ & $B D I$ & 理化评价 & $S P I$ & $B D I$ & 理化评价 & $S P I$ & $B D I$ & 理化评价 & $S P I$ & $B D I$ & 理化评价 \\
\hline $1^{\#}$ & 优 & 优 & I & 优 & 良 & II & 优 & 良 & II & 优 & 优 & II \\
\hline $2^{\#}$ & 优 $^{\mathrm{a}}$ & 优 $^{\mathrm{a}}$ & III & 优 & 优 & II & 优 $^{\mathrm{a}}$ & 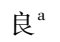 & IV & 良 & 优 & II \\
\hline $3^{\#}$ & 优 & 优 & II & 优 & 优 & II & 优 $^{\mathrm{a}}$ & 优 $^{\mathrm{a}}$ & III & 良 & 优 & II \\
\hline $4^{\#}$ & 良 & 良 & III & 中 & 中 & IV & 良 $^{\mathrm{a}}$ & 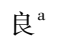 & V & 差 & 差 & 劣 V \\
\hline
\end{tabular}

$\mathrm{a}$ 表示 $S P I$ 或 $B D I$ 和理化评价不一致处.

\section{3 硅藻生态类群组成}

2.3.1 有机污染 Van 等依据硅藻承受有机污染的程度将硅藻划分为 5 个生态类群 ${ }^{[27]}$. 从时间来看 (图 2), 2010

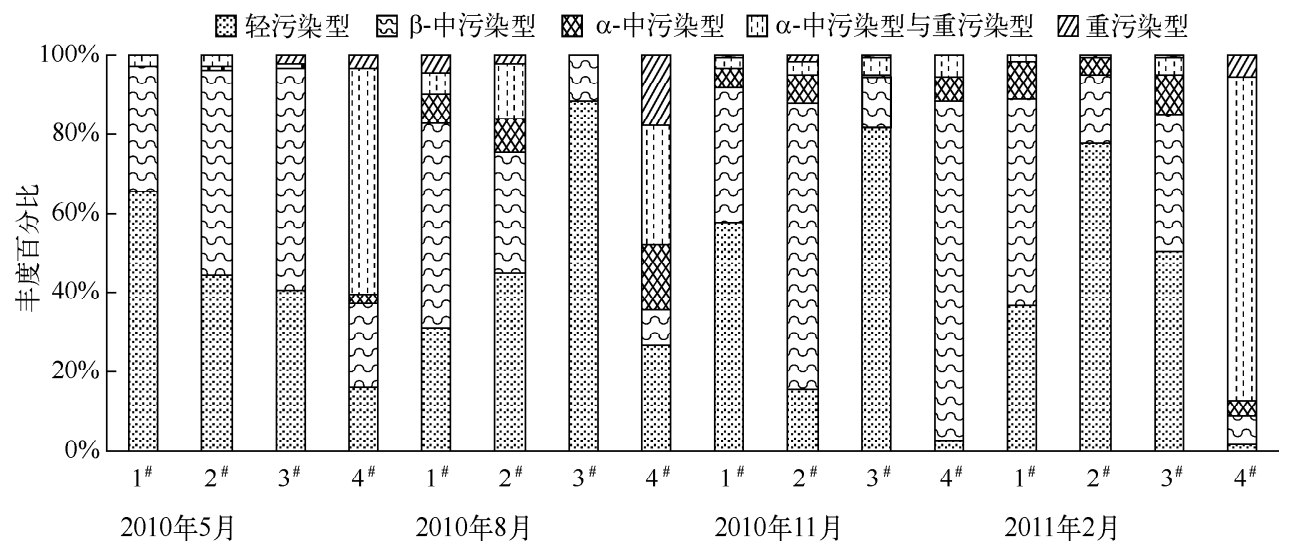

图 2 硅藻有机污染生态类群分布

Fig. 2 Diatoms distribution according to their affinity to organic matter 
年 11 月各断面均以耐低污染种为主,而 2010 年 5 月、2010 年 8 月和 2011 年 2 月除 4 断面外,其他三个断面 亦是以耐低污染种为主. 从空间来看 (图 2), $1^{\#}$ 点、 $2^{\#}$ 点和 $3^{\#}$ 点全年均为耐低污染种 (轻污染型和 $\beta$-中污染 型, 对有机污染物敏感的物种) 占优势, 这与理化监测结果 I、II 类水占 $75.0 \%$ 相吻合; 而 $4^{\#}$ 点于 2010 年 5 月 和 2011 年 2 月以耐中污染与重污染种占优势 (分别为 $60.7 \%$ 和 $87.0 \%$ ), 如 G. parvulum 和 N.palea.

2.3.2 N-异养 N-自养种只能在低有机氮环境下生存; 耐 N-自养种能在某些情况下容忍一定浓度的有机 氮; 兼性 N-异养种需要周期性提高有机氮浓度; 专性 N-异养种需要不断提高有机氮浓度 ${ }^{[27]}$. 从时间来看 (图 3),2010 年 11 月,4 个断面均以自养硅藻为主,而 2010 年 5 月、2010 年 8 月和 2011 年 2 月除 $4^{\#}$ 断面 外, 其他三个断面亦是以自养硅藻为主. 从空间上看 (图 3), 前三个断面均以自养硅藻占优势, 说明前三个断 面的有机污染物浓度为 0 或很低,水质状况较好. 和前三个断面均不相同的 $4^{\#}$ 断面在 2010 年 5 月、2010 年 8 月和 2011 年 2 月以异养硅藻为主 (分别为 61.4\%、51. 0\% 和 86.7\% ), 如 Fistulifera saprophila 和 Mayamaea permitis.

表 2 硅藻指数与各种环境因子之间的皮尔逊相关系数

Tab. 2 Pearson correlation coefficients between diatom index and the environmental factors

\begin{tabular}{|c|c|c|c|c|c|c|c|c|c|c|c|c|c|c|c|}
\hline & SPI & $B D I$ & WT & $\mathrm{pH}$ & DO & $C O D_{M n}$ & $\mathrm{BOD}_{5}$ & Cond & $\mathrm{TP}$ & $\mathrm{TN}$ & $\mathrm{NH}_{4}^{+}-\mathrm{N}$ & Chlo & $\mathrm{NO}_{3}^{-}-\mathrm{N}$ & $\mathrm{NO}_{2}^{-}-\mathrm{N}$ & SDP \\
\hline$B D I$ & $0.92^{* * *}$ & & & & & & & & & & & & & & \\
\hline WT & -0.07 & -0.14 & & & & & & & & & & & & & \\
\hline $\mathrm{pH}$ & 0.27 & -0.02 & 0.24 & & & & & & & & & & & & \\
\hline DO & -0.16 & -0.34 & -0.24 & $0.53^{*}$ & & & & & & & & & & & \\
\hline $\mathrm{COD}_{\mathrm{Mn}}$ & -0.32 & $-0.51^{*}$ & 0.11 & 0.47 & 0.45 & & & & & & & & & & \\
\hline $\mathrm{BOD}_{5}$ & -0.05 & -0.29 & -0.04 & 0.26 & 0.36 & 0.36 & & & & & & & & & \\
\hline Cond & $-0.53 *$ & $-0.63^{* *}$ & -0.31 & -0.04 & 0.55 * & 0.40 & 0.36 & & & & & & & & \\
\hline $\mathrm{TP}$ & $-0.57^{*}$ & $-0.64^{* *}$ & -0.31 & 0.02 & 0.47 & 0.36 & 0.19 & $0.89^{\text {**** }}$ & & & & & & & \\
\hline $\mathrm{TN}$ & -0.44 & $-0.53^{*}$ & -0.43 & -0.10 & 0.47 & 0.28 & 0.36 & $0.96^{\text {**** }}$ & 0.88 & & & & & & \\
\hline $\mathrm{NH}_{4}{ }^{+}-\mathrm{N}$ & $-0.54 *$ & $-0.63^{* *}$ & -0.27 & 0.04 & 0.39 & 0.37 & 0.32 & $0.85^{* * * *}$ & 0.96 & ${ }^{*} 0.89^{* * * *}$ & & & & & \\
\hline Chlo & $-0.66^{* *}$ & $-0.78^{* * *}$ & * 0.09 & -0.04 & 0.40 & 0.49 & 0.42 & $0.87^{* * *}$ & $0.78^{* * *}$ & $*^{*} 0.81^{* * * *}$ & $* 0.77^{* * * *}$ & & & & \\
\hline $\mathrm{NO}_{3}^{-}-\mathrm{N}$ & -0.08 & -0.08 & -0.58 * & $*-0.35$ & 0.30 & -0.06 & 0.11 & $0.68^{* *}$ & 0.42 & $0.71^{* *}$ & 0.34 & 0.43 & & & \\
\hline $\mathrm{NO}_{2}^{-}-\mathrm{N}$ & -0.47 & $-0.62^{* * *}$ & $0.51^{* *}$ & $* 0.15$ & 0.38 & 0.36 & $0.59^{*}$ & 0.32 & 0.12 & 0.18 & 0.13 & $0.60^{*}$ & -0.04 & & \\
\hline $\mathrm{SDP}$ & -0.45 & $-0.62^{* *}$ & 0.21 & 0.13 & 0.33 & 0.44 & $0.75^{*}$ & 0.58 & 0.46 & $0.57^{*}$ & $0.61^{*}$ & $0.76^{* *}$ & $* 0.09$ & $0.74^{* *}$ & \\
\hline SDSi & 0.19 & 0.07 & $0.71^{* *}$ & $* 0.36$ & -0.01 & 0.01 & $-0.29-$ & -0.30 & -0.29 & $-0.43-$ & -0.34 & -0.05 & -0.42 & 0.27 & -0.08 \\
\hline
\end{tabular}

*表示 $P<0.05, * *$ 表示 $P<0.01, * * *$ 表示 $P<0.001$, 达到显著水平以粗体表示.

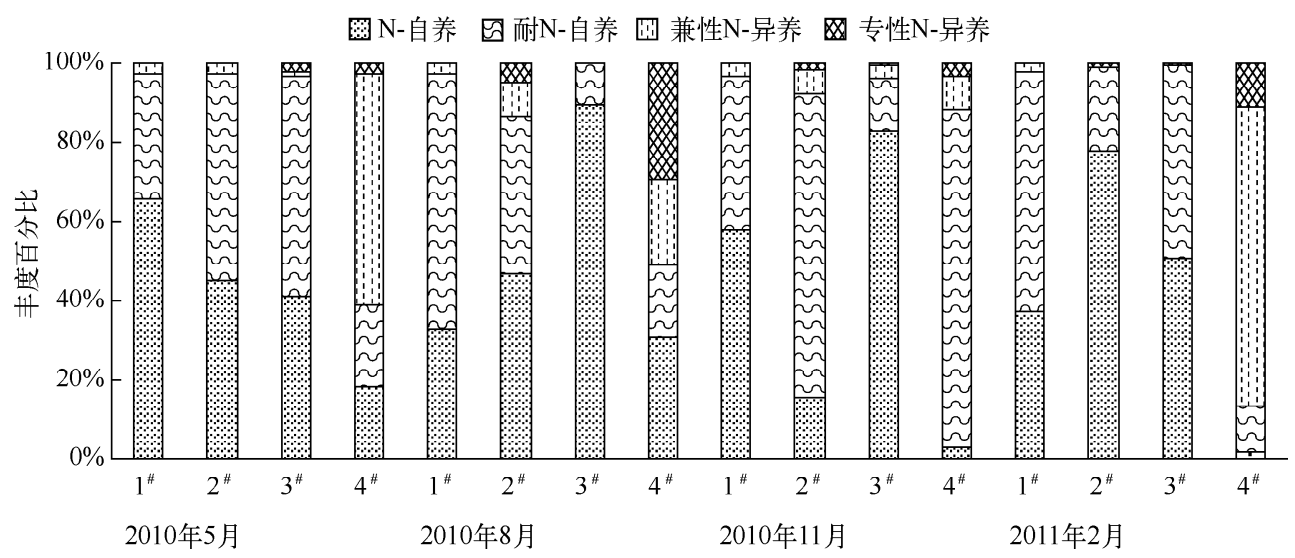

图 3 异养硅藻生态类群分布

Fig. 3 Diatoms distribution according to their heterotrophic capacity 
2.3 .3 氧饱和度 从时间来看 (图 4),2010 年 11 月均以喜好很高氧饱和度种群为主, 而 2010 年 5 月、2010 年 8 月和 2011 年 2 月除 $4^{\#}$ 断面外, 其他三个断面亦是以喜好很高氧饱和度种群为主. 从空间来看 (图 4), 前 三个断面的优势硅藻种均喜好很高氧饱和度 (100\%), 绝大多数断面 (除 2011 年 2 月, $1^{\text {\# }}$ 断面) 未检出喜好 很低氧饱和度 $(<10 \%)$ 的硅藻种群, 说明前三个断面水质优, 水体溶解氧含量较高, 故硅藻以喜好很高氧饱 和度种群占优势. $4^{\#}$ 断面 2010 年 5、8、11 月, 喜好低氧 $(30 \% \sim 50 \%)$ 的硅藻种群依次降低, 分别为 $60.3 \%$ 、 $43.6 \%$ 和 $5.7 \%$; 到了 2011 年 2 月, 喜好低氧 ( $30 \% \sim 50 \%$ ) 的硅藻所占比例急剧增加 ( $86.5 \%$ ), 说明水体中 溶解氧含量下降较大,水质状况下降,这与同期理化水质评价为劣 $\mathrm{V}$ 类相吻合.

图很高四高 四中等因低 四很低

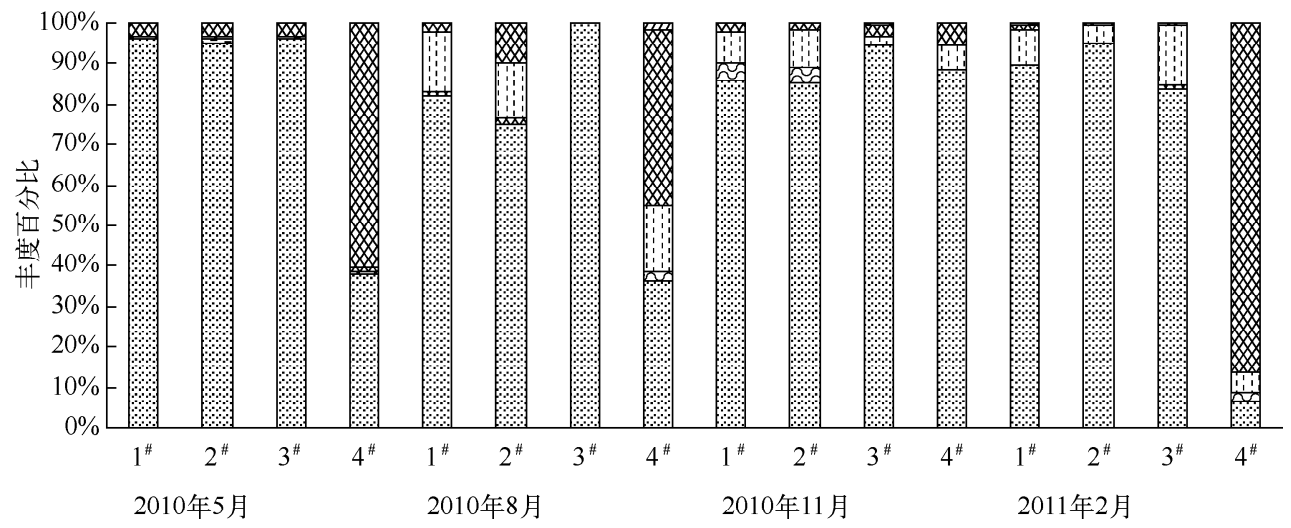

图 4 好氧硅藻生态类群分布

Fig. 4 Diatoms distribution according to their affinity to dissolved oxygen

$2.3 .4 \mathrm{pH}$ 偏好 从整体上看, 全年水体均以喜中性和碱性的硅藻类群为主, 这与同期调查白沙溪 $\mathrm{pH}$ 均值为 $7.29 \pm 0.61$ 相吻合. 从时间来看 (图 5),2010 年 5 月和 8 月,各断面均以喜中性硅藻占优势;2010 年 11 月, $1^{\#}$ 和 $4^{\#}$ 断面以喜中性硅藻为主, $2^{\#}$ 和 $3^{\#}$ 断面以喜碱性硅藻为主;2011 年 2 月, $1^{\#}$ 和 $3^{\#}$ 断面以喜中性硅藻为 主, $2^{\#}$ 断面喜中性硅藻和喜碱性硅藻各自所占比例相当 ( $50.9 \%$ 和 $46.6 \%$ ), 而 4 断面以喜碱性硅藻占绝对 优势 ( $85.2 \%$ ). 从空间来看 (图 5), $1^{\#}$ 断面以喜中性硅藻为主; $2^{\#}$ 和 $3^{\#}$ 断面, 除 2010 年 11 月外, 均以喜中性 硅藻为主; 4 \#断面 2010 年 5 月至 2010 年 11 月均以喜中性硅藻为主, 2011 年 2 月以喜碱性硅藻为主.

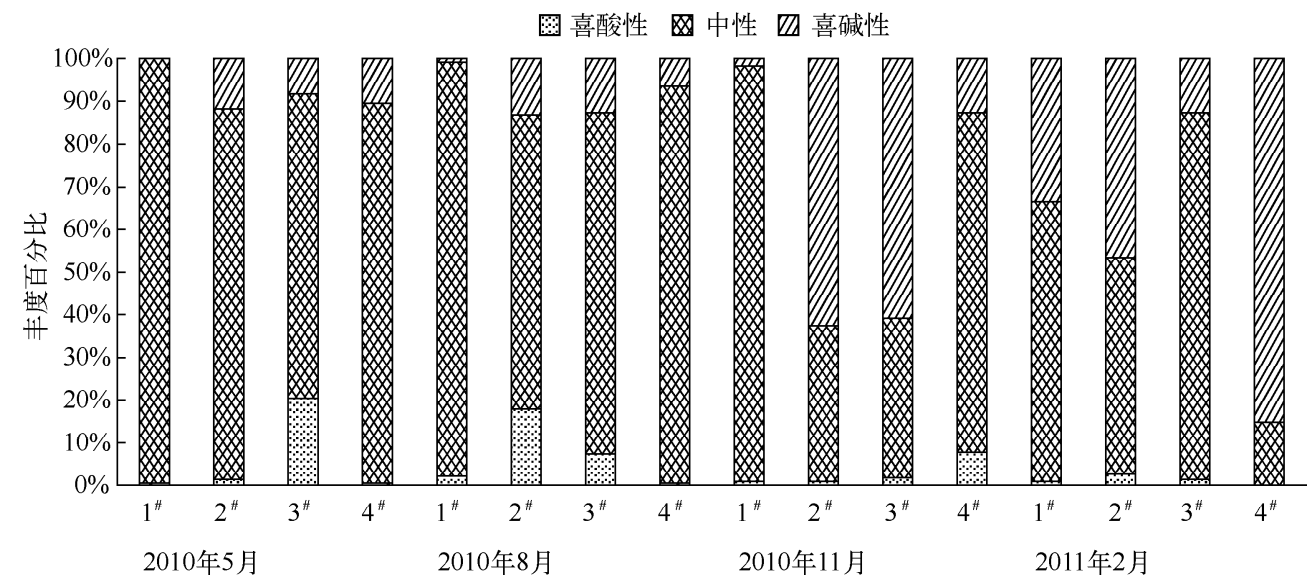

图 5 喜好不同 $\mathrm{pH}$ 硅藻生态类群分布

Fig. 5 Diatoms distribution according to their affinity to $\mathrm{pH}$ 


\section{$2.4 S P I 、 B D I$ 指数与理化参数相关性分析}

$S P I$ 与电导率、总磷、铵氮、氯化物之间呈显著负相关; $B D I$ 与高锰酸盐指数、电导率、总磷、总氮、铵氮、 亚硝酸盐、可溶性磷酸盐呈显著负相关, 与氯化物之间呈极显著负相关 $(P<0.001)($ 表 2).

\section{3 讨论}

本研究通过对硅藻 SPI、BDI 与水质理化指标的比较与分析, 发现对于不同的监测断面, 水质理化评价 和硅藻 $S P I$ 及 $B D I$ 生物评价存在一定的差异, 这是因为水质理化评价为瞬时值 (不稳定), 以各种检测指标 中超标最严重的因素确定水质类别, 而硅藻生物评价则反映了一定时间各种环境因素对于水生生物综合作 用的结果. 这与赵湘桂等 ${ }^{[12]}$ 利用硅藻 $S P I 、 B D I$ 和水质理化指标评价漓江水质所得结果相同. 本研究采用 OMNIDIA 软件计算 SPI 和 $B D I$ 值是基于欧洲河流硅藻生物监测方法, 其在中国境内不同河流的适用性则需 开展进一步的研究: 如(1)开展更大流域尺度和时间尺度的取样工作; (2)比较 OMNIDIA 软件中硅藻赋值与在 中国境内不同气候条件下各流域硅藻赋值的异同, 开发完全适用中国各流域的 OMNIDIA 软件; (3)完善硅藻 $S P I$ 指标、BDI 指标与理化指标评价结果对接的科学合理性.

理化评价中参与评价的项目包括 $\mathrm{pH} 、 \mathrm{DO} 、 \mathrm{COD}_{\mathrm{Mn}} 、 \mathrm{BOD}_{5} 、 \mathrm{NH}_{4}^{+}-\mathrm{N} 、 \mathrm{TP}$, 其中 $\mathrm{TP}$ 和 $\mathrm{NH}_{4}^{+}-\mathrm{N}$ 均与 $S P I 、 B D I$ 呈显著负相关, $\mathrm{COD}_{\mathrm{Mn}}$ 与 $B D I$ 呈显著负相关, 这也从侧面说明了 $S P I$ 和 $B D I$ 水质评价结果的有效性.

从本研究结果硅藻生态类群组成中可以看出, $4^{\#}$ 断面除 2010 年 11 月外, 均以耐中污染与强污染硅藻、 异养硅藻和喜好低氧硅藻类群均占优势, 同期理化指标水质评价结果亦较差, 为 III 类 (2010 年 5 月)、IV类 (2010 年 8 月) 和劣 $\mathrm{V}$ 类 (2011 年 2 月), 这可能与 $4^{\#}$ 断面上游 $50 \mathrm{~m}$ 处工厂工业废水以及白龙桥镇生活污水 的双重污染有关. 建议有关部门加强对白沙溪 $4^{\#}$ 断面沿岸工业废水和生活污水的监管.

致谢: 感谢法国 Asconit 公司 Berengere LASLANDES 博士和 Roser ORTIZ 博士协助鉴定硅藻种类; 感谢法国 Asconit 公司 Berengere LASLANDES 博士在数据处理分析上给予的帮助和支持; 感谢金华市水文站黄忱、曹 樱樱、蒋川和黄可谈提供白沙溪水质理化数据.

\section{4 参考文献}

[ 1 ] 胡鸿钧,魏印心. 中国淡水藻类. 北京:科学出版社,2006:300-303.

[ 2 ] Battarbee R, Flower RJ, Juggins $\mathrm{S}$ et al. The relationship between diatoms and surface water quality in the Hoylandet area of Nord Trondelag, Norway. Hydrobiologia, 1997, 348 : 69-80.

[ 3 ] Van DH. Partial recovery of moorland pools from acidification: indications by chemistry and diatoms. Aquatic Ecology, 1997,30 : 203-218.

[ 4 ] Kelly MG. Use of the trophic diatom index to monitor eutrophication in rivers. Water Research, 1998, 32: $236-242$.

[ 5 ] Potapova MG, Charlesd F. Benthic diatoms in USA rivers: distributions along spatial and environmental gradients. Journal of Biogeography, 2002, 29: 167-187.

[ 6 ] Kelly MG, Penny CJ, Whitton BA. Comparative performance of benthic diatom indices used to assess river water quality. Hydrobiologia, $1995,302: 179-188$.

[ 7 ] Lenoir A, Coste M. Development of a practical diatom index of overall water quality applicable to the French National Water Board network. In: Whitton BA, Rott E eds. Use of algae for monitoring rivers II. Institut für Botanik, Universität Innsbruck, 1996 : 29-43.

[ 8 ] Schiefele S, Schreiner C. Use of diatoms for monitoring nutrient enrichment acidification and impact salts in Germany an Austria. In: Whitton BA, Rott E, Friedrich G eds. Use of algae for monitoring rivers. Institut für Botanik, Universität Innsbruck, 1991.

[ 9 ] Prygiel J, Lévéque L, Iserentant R. Un nouvel indice diatomique pratique pour l'évaluation de la qualité des eaux en réseau de surveillance. Revue des Sciences de l'eau, 1996, 9(1):97-113.

[10] Watanabe T. Numerical simulation of organic pollution in flowing waters. In: Encyclopaedia of environmental control technology Vol. 4: Hazardous waste containment and treatment. Houston: Gulf Publishing Company, 1990: 251-281.

[11] Kelly MG, Whitton BA. The trophic diatom index: A new index for monitoring eutrophication in rivers. Journal of Applied Phycology, 1995, $7: 433-444$. 
[12] 赵湘桂, 蔡德所, 刘 威等. 漓江水质硅藻生物监测方法研究. 广西师范大学学报: 自然科学版, 2009,27 (2): 142-147.

[13] Dela-Cruz J, Pritchard T, Gordon G et al. The use of periphytic diatoms as a means of assessing impacts of point source inorganic nutrient pollution in south-eastern Australia. Freshwater Biology, 2006, 51 : 951-972.

[14] Goma J, Rimet F, Cambra J et al. Diatom communities and water quality assessment in Mountain Rivers of the upper Segre basin (La Cerdanya, Oriental Pyrenees). Hydrobiologia, 2005 , 551 : 209-225.

[15] Wang YK, Stevenson RJ, Metzmeier L. Development and evaluation of a diatom-based index of Biotic Integrity for the Interior Plateau Ecoregion, USA. Journal of the North American Benthological Society, 2005, 24: 990-1008.

[16] 陈 旭,羊向东, 董旭辉等. 近 50 年来环境变化对巢湖硅藻组合演替的影响. 湖泊科学, 2011,23(5):665-672.

[17] 董旭辉, 羊向东, 王 荣等. 长江中下游湖泊硅藻-总磷转换函数. 湖泊科学, 2006,18(1):1-12.

[18] 王翠红, 张金屯. 汾河水库水源河着生硅藻群落的 DCCA 研究. 中国环境科学, 2004,24(1):28-31.

[19］董旭辉,羊向东,王 荣. 长江中下游地区湖泊富营养化的硅藻指示性属种. 中国环境科学,2006,26(5):570-574.

[20] 郭 云, 赵宇中, 张 波等. 乌江中上游水体营养状况底栖硅藻指示性属种的研究. 中国环境监测, 2010,26 (6) : 80-84.

[21] Tang T, Cai QH, Liu JK. Using epilithic diatom communities to assess ecological condition of Xiangxi River System. Environmental Monitoring and Assessment, 2006, 112 :347-361.

[22] Asconit Consultants. EN 13946:2003, NF T90 357 1, guidance standard for the routine sampling and pretreatment of benthic diatoms from rivers. Lyon, 2003, Agence de l'Eau Rhǒne.

[23] Asconit Consultants. AFNOR NF T90-344, determination de l'index poissons rivière ( IPR). Lyon, 2004, Agence de l'Eau Rhǒne.

[24] 长江流域水环境监测中心. 水环境监测规范(SL219-98). 北京: 中国水利水电出版社, 1998 .

[25] Lecointe C, Coste M, Prygiel J. 'Omnidia' : Software for taxonomy, calculation of diatom indices and inventories management. Hydrobiologia, 1993, 269/270:509-513.

[26] 国家环境保护总局, 国家质量监督检验检疫总局. 地表水环境质量标准 (GB3838-2002). 北京: 中国环境科学出 版社, 2002 .

[27] Van DH, Mertens A, Sinkeldam J. A coded checklist and ecological indicator values of freshwater diatoms from the Netherlands. Aquatic Ecology, 1994, 28(1):117-133. 